\title{
Cultural Diversity and Quality Care in Lebanon
}

\section{Fadi Abou-Mrad ${ }^{1,2,3,4 *}$ and Lubna Tarabey}

${ }^{1}$ Head Neurology Division, Saint Charles Hospital, Lebanon

${ }^{2}$ Division of Neurology, Faculty of Medical Sciences, Lebanese University, Lebanon

${ }^{3}$ Head Division of Medical Ethics, Faculty of Medical Sciences, Lebanese University, Lebanon

${ }^{4}$ Laboratory of Medical Ethics and Legal Medicine-University Paris Descartes, Paris

${ }^{5}$ Department of Sociology, Faculty of Medical Sciences, Lebanese University, Lebanon

\begin{abstract}
The quality of medical care given to any patient is largely dependent on the treatment of an individual as a whole-a treatment that would cater to the physical, psychological, spiritual and mental aspects. In Lebanon, the medical field seems to be ignorant to this reality and continues to offer treatment that ignores the unity of the human person.

The objective of this study is to provide an insight to the importance of reintegrating the human person in the medical field, a process that requires the contribution of different support systems associated with health care. This would bring the topic into public dialogue allowing for a reassessment of the issue. The method utilized included semi structured interviews, seminars and open discussion panels that included a myriad of Lebanese religious authorities, physicians, legislators, parliament members and social activists. The outcome of these interviews and discussion revealed a contradiction between the ideological understanding of the importance of the value of the human person and the actual or practical application of this value. Most people who participated in the study acknowledged the importance of catering to the human in suffering as a whole focusing on the physiological aspect of illness but equally so on the spiritual, psychological and mental. However, those same people acknowledged that the situation in Lebanon was not considerate to the principle of unity of human person. The reasons for this discrepancy had their socio-economic and cultural roots and hindered the quality of care delivered to suffering individuals. This discrepancy cannot be overcome or ratified except when all those concerned with medical care acknowledge the problem and show a willingness to deal with it through taking positive action to rectify the situation.
\end{abstract}

Keywords: Human person; Quality of care; Cultural diversity; Lebanon; Medical ethics

\section{Introduction}

Lebanon, a multi sectarian country, has suffered from an extensive civil war that had left its impact on all the country's social institutions and has consequently marginalized the human being. This marginalization is perhaps mostly felt within the medical domain where a lack of application of the concept of the human person seems to characterize it. The human person is a concept that upholds the true quintessence of the human being and, with in the field of medicine, finds its application in the perpetuation of the integrity and unity of the human being allowing for the treatment of an individual as a whole-physical, psychological, spiritual and mental ${ }^{[1]}$. For a true and comprehensive healing process to occur the unity of the human being must not be jeopardized but a remedy that targets both body and soul must be devised. Contemporary medicine is realistically aware of the connection and has as such devised a novel medical pedagogy that specifically targets that aspect [1]. The current problem is how to think consent release that underlies and expresses the principle of autonomy in order to neutralize paternalism in a society pushing to the extreme the notions of risk, precaution and responsibility. Therefore, a multi disciplinary approach aiming at acculturating medicine for social cohesion is becoming indispensible. This needs an ethical approach that is a guide for the quality of care emphasizing patient participation, competency, and technical excellence, methods of assessment improvement and respect of vulnerability keeping in mind that these elements are cumulative and never dissociated.

${ }^{1}$ This perception was understated by Plato who considered that a doctor must offer a treatment that targets the patient as a whole and not just heal the sick organ or symptom-the soul and the body must be targeted. For more see Real Giovanni, 2002.
The problematic situation that faces the medical profession in Lebanon directly emanates from a contradiction between ideology and practice. On a cultural level, all sectarian groups in Lebanon as represented by religious authorities agree that a realization of human integrity and unity constitutes the basis for the establishing of a better, healthy society. On a practical level, and within the field of medicine, health care practitioners also agree on the importance of the integrity of the human person but when it comes to actual practice, researchers have noted an astonishingly lack of adherence to the value of integrity and unity of the human person. One particular research done by one of the article's authors, carried out on a national level in Lebanon and targeting nurses as medical care providers in hospitals revealed a lack of understanding of the values of respect of the human person and social solidarity among $95 \%$ of the nurses understudy [2]. In fact only, $2.5 \%$ of the nurses could offer a proper definition of human dignity, the respect of man in every man. Most shockingly, $66 \%$ rejected the value of vulnerability, as physically or mentally fragile patients under the burden of psycho affective susceptibility or added risk, brushing it aside as unimportant or inconsequential to the medical care. They were not able to realize that physically and mentally fragile individuals need protection (ex: vegetative coma, motor deficit, pregnancy, chronic or

*Corresponding author: Fadi Abou-Mrad, Assistant Professor, Department of Neurology and Medical Ethics, Lebanese University, Lebanon, Tel: +9613392 600; E-mail: fadiaboumrad@gmail.com

Received August 14, 2012; Accepted November 09, 2012; Published November 28,2012

Citation: Abou-Mrad F, Tarabey L (2012) Cultural Diversity and Quality Care in Lebanon. J Clinic Res Bioeth 3:140. doi:10.4172/2155-9627.1000140

Copyright: ( 2012 Abou-Mrad F, et al. This is an open-access article distributed under the terms of the Creative Commons Attribution License, which permits unrestricted use, distribution, and reproduction in any medium, provided the original author and source are credited. 
mental disease, etc). Furthermore, the ability to properly distinguish the concepts of ethics-the judgment of value of health care decisions and deontology-the duty of those concerned with health care-was not reflected in $73 \%$ of the nurses. This overall failure on the part of the nurses to identify ethics as an emerging multi-disciplinary science reflects the situation of the medical field. The human being with value and dignity has been eradicated from the general portrait of the patient-health profession relationship [2].

For an improvement in the quality and efficiency of medical care, a reintegration of the person into its body must be attempted mainly through reestablishing within the field of medicine the values of social solidarity that are the cardinal values that build a health system. Which can only be achieved through appropriate realization of determining indicators. These indicators are respect of human dignity, awareness of vulnerability, participation of patients, and the availability of competent human resources and technicality which is fundamental for quality care ${ }^{[2]}$.

The aim of this paper is to provide insight into the importance of reintegrating human dignity, deontology, value and ethics as a guide line for quality in the medical profession, through bringing into the limelight the dissociation between the practice and the theory in a culturally diverse society like Lebanon. Evidently, this would not be possible without engaging the different support systems associated with health care-namely social security systems, ministry of health, public sector, private sector, insurance companies and non-profit institutions, mostly handled by the religious communities. Furthermore, a general legislative text outlining medical practices and biomedical laws must be properly formulated, put in practice and continuously revised. Examining the lack of application of bio-medical laws accompanied by the lack of concern for human integrity bring the matter into public discourse allowing for a reconsideration of the situation.

\section{Method}

Carrying out a comprehensive literature review on the topic revealed a lack of interest among scholars in addressing from a sociomedical point of view, the issue of the human person with the medical profession. The patient continues to be dealt with as a medical case, not as a complete human being with integrity. This intensified the need for a research that would reveal the reality of the situation from a medical but also a cultural perspective. Such a research, that attempts to bring together several disciplines, is the first of its kind. The main method utilized included semi-structured interviews, seminars and open discussion panels. A total of fifteen professionals including religious authorities, ministers, physicians, legislators and parliamentary members were interviewed. All these people are fundamental for the development and establishment of a bio-medical law. In Lebanon, personal status affairs ${ }^{[3]}$ are the main concern of the religious sects and any law requires the approval of these leaders; hence their inclusion was essential in the interviews as they reflect the culture of the country.

${ }^{2}$ Providing technologies is important as that allows for the use of appropriate tools for patient care. However, human technicality and know how is also requiredtechnicality that allows for the suitable use of these technologies and their maintenance. As Fouad Boustani puts it "Civilization is not to asphalt the road but to know how to maintain it [6]." For example, technologies are available in Lebanon, but maintenance and electricity is not which creates a hindrance in the face of the physician's capacity to provide appropriate medical care.

${ }^{3}$ Personal matters including inheritance, marriage, divorce, custody, support and maintenance are dealt with in religious courts. Each sect in Lebanon has its own court. Hence, any law that touches on the personal status such as custodianship is in the hands of religious leaders who constantly need to be approached whenever a new law is to be proposed.
The semi structured interviews were considered the most useful technique for data gathering as they allowed for an expressive reflection on and of the perspectives of key players in the establishment of a quality care system in Lebanon. The interviews provided qualitative data that reflected the interviewees reflections on the following topics: their envision of the human person; the variables that modify the concept of the human person in Lebanon; the religious dependency of Lebanese people that hinder the realization of the human person; obstacles, including social and economical, that hinder the realization of the integrity of the human person and the legislation of a bio-law; their opinions regarding placebo trials; problems of gender inequality and ethics in Lebanon.

In addition to semi structured interviews, a total of six panels and three seminars bringing together a number of experts from the different faculties of science, medicine, dentistry, pharmacy, health care, engineering, law and social sciences from the nation university in Lebanon-the Lebanese University which caters to the majority of students in Lebanon. The Lebanese University, as such, reflects the cultural diversity of the Lebanese society at large. These served as backbones against which the data collected through interviews were placed and accordingly analyzed.

\section{Results and Discussion}

The interviews and discussions revealed a discrepancy between ideological and theoretical perspective of the value of the human person and the current manifestation of this value in reality.

\section{The human being in the culture}

Christian religious personalities interviewed all seemed to uphold a similar point of view-namely that the human being is one unity of body and spirit. The spirit lives in this unique body and is in communion with it which is why Christians believe in the resurrection of the chair which symbolizes the restoration of human unity [3]. However, Khodr emphasized that Lebanon, from its early birth in 1920, was composed of confessional communities and never a group of responsible persons. "There is no sensitivity to patriotism, to the community of men, independently from religious beliefs"

In Islam, too, the emphasis on the respect of the human being as one whole- a unity is characteristic of the Fiqh and the Sharia'. The preservation of the human body and the safe guarding of human integrity are at the basis of Quranic teaching. (ref. $95^{\text {th }}$ sura, the Fiqh $4^{\text {th }}$ verse). Late Fadlallah, a prominent Islamic figure, assured in an interview in 2008 that human beings should abide by the principles of ethics and responsibility towards God and other fellow humans. Nevertheless, he assured that the Lebanese individual is shaped by the events that influence the individual's own sectarian group leaving major impact on his thoughts and behavior. This contradiction between ideology and reality leads to a dualism in people's personalities [4].

The former president of the Parliamentary Commission on Human Rights [5], confirms that the war, continued insecurity and overall Middle Eastern environment led to a negative culture trivializing the physical integrity and values of the human person. He continued that the Lebanese society encompasses too much pain and suffering that a disease, whatever its severity, is also trivialized. He also witnessed to the weakness of the parliament in regulating the social life of the Lebanese people.

The above discussion clarifies that the value attributed to the human being with its accompanying unity and integrity seems to characterize 
the ideological framework. However, these ideas and values are only paid lip service in Lebanon while in reality an ignoring of the human being's unity and integrity seems to be characteristic of the Lebanese contemporary culture. The following part will shed light on the value of the human person within the health field.

\section{The human person in health care professions}

The medical profession in Lebanon is characterized by the marginalization of the human being which was a direct reflection of the political and socio-economic instability in the country during and after the civil war. The sick individual during times of instability is largely dehumanized. A patient becomes an individual with a sick body that needs medical treatment that ultimately targets the disease and not the human being as a whole. This was the result of the change in medical practice that, according to the general secretary of the National Committee on Bioethics, Professor Fouad Boustany [6], has lost its essential vocation. He claims, "That the physician is carrying his or her culture and environment into the clinic." This is largely due to many variables including the war, dissociation from medical ethics and deontology, and the heterogeneity of the medical body due to physicians studying abroad under different educational systems, the augmentation of the number of physicians and its economical implication, in addition to the confessional influence on the Lebanese constitution. This has influenced the medical practice rendering it anarchists. This was further strengthened by the absence of national clinical guidelines that are indispensable for the maintaining of the competency, the methods of assessment and improvement of health care professionals.

The social and economical experts input highlighted the facts that led to the modification of the relationship between the different partners of the health care system. These include the universities, syndicates of hospitals, the Lebanese order of physicians, other health care professionals and the scientific societies. The head of the judicial department at the Lebanese Order of Physicians Maitre Charles Ghafari confirms that "Lebanese Medicine and hospitals are a simplified expression of the Lebanese Reality".

The factors that were mentioned include the persistence of economical difficulties, the extensive technicality of the hospital and the institutional non-credibility of sciences coupled by the non-credibility of physicians who prescribe complementary non indicated tests all left a negative impact on the human person.

Charles Ghafari [7] confirmed also that "the medical secrecy is very fragile because the private life is not respected". This is characteristic of the Lebanese society and of the nature of family life that is largely extended in reality and is reflected in the collectivity of relatives that always surrounds a patient ${ }^{[4]}$. This fact affects the principle of confidentiality that is important in a quality health care decision making process [8].

Furthermore, the principles of truth telling and patient's autonomy are also disregarded. This fact was strongly supported by a national survey confirming that consent release is absent in around $83 \%$ of Lebanese hospitals. Among the $17 \%$ of the hospitals conducting patient consent, the half of these revealed a weakness in implementation of the policy and the method of acquiring patient participation [9].The patient is constantly approached with a 'paternalistic' attitude [10] not asked to share in the decision making process that directly concerns his/her health. Doctors adopt this paternalistic attitude assuming either that they are protecting their patients or that their patients don't need

${ }^{4}$ For more on the topic see Abou Mrad et al., 2012. to know all details "Patients have the full right to receive appropriate time from physicians to be more informed about their health and therapeutic options "[7], reflecting a total disregard for the basic rules of ethics.

The instability within the Lebanese society is definitely an important variable for the lack of concern for the human person but the nature and application of the scientific objective method is also responsible for the situation. The medical profession in Lebanon is characterized by the adoption of the principles of rationality, objectivity and neutrality (Elliot, 2008). These principles, in themselves, are not wrong but when they become the only principles applied, dissociation between the treatment and the human being results. A patient is perceived as a sick individual and a disregard of the human being within is practiced.

Lebanon is a relatively small country with a population of 3.5 million people but the physician to citizen ration is quite remarkable: 248 physicians for every hundred thousand citizen (World Population Prospects: The 2008 Revision). Thus, the availability of physicians is not a problem. On another level, and particularly after the end of the war, technical availability ceased to be a problem as well. Medical equipment is available at a very high standard allowing for an acceptable level of medical treatment. However, the main problem in Lebanon lies not in the availability of technologies and scientific expertise but in the lack of sensitivity and concern for the human person. The problem is mainly ethical in nature. There is an astonishing lack of concern, within the medical profession, with the patient as a human being and not just as an individual with an illness. The ministry of health and the role it plays is important since it is the main state agent concerned with health care. "It is becoming mandatory to legislate in Biolaw and allows the ministry of Health to fulfill its role not only through the employees but also with the concerned syndicates, universities and hospitals under the condition of being supervised by the ministry of health that continue playing a major role in this field"[11].

Another problem with the quality of care in Lebanon is economical in nature. Medical care is largely covered by the Ministry of Public Health which although recently slightly reviewed its tariff continues to delay payments to hospitals and physicians for as long as two years. This creates an economical strain on both which they resolve through increasing the fees required from private patients and increase the number of required tests. The former president of the Lebanese Order of Physicians Georges Aftimos states that "I am wondering that this test abuse in Lebanese medical profession is disregarding the integrity of the patient who is subject to tests that are not always essential for medical care" [12].

The rich in Lebanon with full medical coverage from private insurance companies are favored by both hospitals and physicians as they provide a sustained income. This social strata provides the income for medical care institutions and hence receive the medical care needed. However, once again, the vulnerable elements of the Lebanese population are excluded because they are not able to afford medical care. Nevertheless, the mostly abused strata are those without any sort of medical coverage and are referred to as self payers. These self payers are largely abused and made to pay beyond what is required or necessitated by their medical condition. Test abuses in these cases become phenomenal.

All this coupled with the absence of concern with consent and meaning of vulnerability has left its impact on the value of medical care delivered to a human being and not to a sick case. 


\section{Conclusion}

\section{Reestablishing the human person in medicine}

The above argumentation and discussion clearly reveals a sever lack in the determinants of the quality of care and the cardinal values that found a health system.

How can the medical profession overcome this predicament?

The first and foremost line of action rests in assuming responsibility for one's actions and choices. French (1992) clarifies that although most people spend considerable time and effort trying to avoid responsibility for any action or situation. Yet, the only way out of this predicament lies in assuming rather than avoiding responsibility. The doctors, legislators, medical institution and the civil society at large must take positive action and assume responsibility seeking to change the current situation. Unless such a step is taken, and as long as attempts at blaming others - be it the war, politicians, capitalist order or other-continue to exemplify the domain, change and a realization of the value of the human person would not be possible.

Doctors play an essential role in the restoration of the human being within the medical profession. This becomes easy to achieve if doctors train themselves to listen to their patients and to acknowledge all different aspects of the human-not just focus on the physical or biological. Charon [13] claims that doctors "recognize symptoms, emergencies and the need for action. If we complete this with recognition of the people in the midst of their distress, we become better complete doctors." Evidently, we are not asking doctors to ignore the biological and evidence based medicine but to acknowledge the psychological and spiritual. Finally, we must acknowledge that what applies to doctors also applies to all those associated with care giving including nurses and other health professional.

Medicine and health care professionals should become a corpus exceeding mere knowledge that should take into consideration the legal and social context before defining and planning therapeutic options. The more sincere the institutions, the more physicians will express loyalty and show professionalism, the more people will trust the system and the more the ethical exigencies will become indispensible to the action of every medical and hospital responsible. All these are unique conditions to increase the efficiency of the health care system. Hence, between health care demands and management, between individuals and collectivity, between patient's rights and liberty of physicians, between the choice of investors and the priority of each, there is a part of medical ethics that is not prevailing in Lebanon and which cannot be realized without business ethics and medias ethics.

\section{References}

1. Llorca G (2003) Guide pratique de la décision médicale: I'éthique en Clinique. Med-Line éd, Paris.

2. Abou-Mrad F, Attallah C, Antoun N, Kerbage M, Melhem G, et al. (2010) Qualité éthique de l'exercice de la profession d'infirmier au Liban. Therapie 65: 551-565.

3. Khodr G (2008) Orthodox Bishop of Mount Lebanon. Interviewed by Fad Abou-Mrad on September 6.

4. Fadlallah, Hussein M (2008) Ulema Chiite in Lebanon. Interviewed by Fad Abou-Mrad on September 3.

5. Mokheiber G (2008) Parliament Commission on Human Rights. Interviewed by Fadi Abou-Mrad on August 30.

6. Boustany F (2008) Genral Secretary of National Committee on Bioethics Interviewed by Fadi Abou-Mrad on August 28.

7. Ghafari C (2008) Interviewed by Fadi Abou-Mrad on August 28.

8. Husted GL, Husted JH (1991) Ethical Decision Making in Nursing. Mosby Year Book, Michigan.

9. Abou-Mrad F (2010) Le Cadre Législatif de essais Clinique au Liban, Review Med Brux 31: 467-473.

10. Noujeim C, Mourad C (2011) Substitue decision making in Lebanese ICU: are we protecting the vulnerable?, Thesis submitted to the Lebanese University, School of Medicine.

11. Khalife, Jawad M (2008) Former Lebanese Minister of Health. Interviewed by Fadi Abou-Mrad on September 10.

12. Aftimos G (2008) Former president of the Lebanese Order of Physicians. Interviewed by Fadi Abou-Mrad on August 28.

13. Charon R (1998) "To listen, to recognize" in Perspectives in Medical Sociology Phill Brown, Wadsworth Publishing Company, Belmont. 